\title{
Graphic data as object of system analysis
}

\author{
Aleksandr V. Kuchuganov \\ Department of Automated Data Processing and Control Systems \\ Kalashnikov Izhevsk State Technical University \\ Izhevsk, Russian Federation \\ aleks_kav@udm.ru
}

\begin{abstract}
The relevance of the system approach to the problems of graphic data analysis proceeds from the degree of its substantial volume and validity in the information about an object. System analysis methods being abstracting and specialization, structuring and restructuring, recursive specification and iteration proximity advance a set of claims with respect to the methods of analysis and synthesis of graphic data and their interaction. The paper objective is to demonstrate the benefits of the system approach to the graphic data analysis through the example of analysis methodology and aerospace images deciphering.
\end{abstract}

Key words-System analysis, structured analysis, methodology, image processing methods, fuzzy graphs, description logic, ontology, logical conclusion, aerospace image analysis.

\section{INTRODUCTION}

The fast developing computer technology and the diversity of computer products as well as the explosive growth of data flow, including the graphic one, drive the need in systems approach to data analysis. The relevance of systems approach to data processing and analysis derives both from its large volume and validity in the information about an object and in high specialization of the most image recognition techniques.

Traditionally, the tasks of data processing and analysis are aimed to solve the following problems: 1) transmission and processing; 2) image recognition; 3) image analysis.

The tasks of image recognition (enlarging) lie in calculating the measure of proximity between the data points in the parameter space (Bayesian approach), or graph grammar-based approach, which involves matching with the patterns defined as grammars for the constituent elements. However, it is still problematic to recognize conjoined handwritten text, notwithstanding the fact that it contains only 3 basic elements: lines, hooks and loops.

Image analysis differs from image recognition in the same manner as logical thinking differs from the visual one. Analyzing images is the problem of modeling the reasoning on the basis of graph data, for which it is required to retrieve formal description out of the image, to apply the methods of logical inference and patterns matching, in other words there is the need to develop the methods of structural analysis being the major component of system analysis, abstracting and specialization, analysis and synthesis.

The objective of the system approach to graph data analysis is to retrieve practically useful information about the structure and parameters of the objects represented in the images in as precise and full form as possible.

\section{THE PROBLEM OF SYSTEM ANALYSIS AND GRAPH DATA DESCRIPTION}

Global and local descriptors are typically used for describing the images. Global descriptors enable to describe the images' color specifications gained via histogram methods and texture specifications describing homogeneous objects in the image. Local descriptors describe the image segment chosen by a certain way [1]. The most commonly used descriptors are Scale Invariant Feature Transform, Speeded Up Robust Features, Rotation Invariant Fast Features, which are invariant to the scale, rotation, noise and lighting transformation.

Lutsiv V.P. [2] proposes the object independent approach to the structural analysis of images and presents the model of structural organization of the surrounding world at different hierarchical levels varying from the textures miscrolevel to the objects and subobjects macrolevel. The approach involves the object independent structural decomposition, generation of image structural descriptions and object independent method of matching the images of the real scenes on the basis of their hierarchical structural descriptions. Independence from the objects types is the advantage of the approach. Using the opportunity to refer back and reconfirm the solutions under the conditions of semistructured problems only at the stage of descriptions matching can be considered as a disadvantage.

The research of Simankov V.S., Lutsenko E.V. and Laptev V.N. [3] can be considered to be the confirmation of the fact that graph data analysis can comply with the general framework of system analysis. The research under consideration suggests an original approach to the synthesis of adaptive automated systems maintaining control over the complicated systems, which are based on the methods of image recognition and decisions taking. The software package Universal cognitive analytic system "Aidos" implementing the models and technologies suggested by the authors is described in the paper.

The task of matching data presented as graphs was studied under the conditions of problem comprehending and evaluating. Adam Stotz et al. [4] present the situation as Directed Attributed Relational Graphs (DARG), which are simple graphs containing the aggregations of the attributes of the peaks and edges. DARG-graph $\mathrm{G}$ is indicated as $\mathrm{G}=\{\mathrm{V}, \mathrm{E}$, $\mathrm{AV}, \mathrm{AE}\}$, where $\mathrm{V}$ is the aggregate of peaks, $\mathrm{E}$ is the aggregate of edges, $\mathrm{AV}$ is the finite aggregate of peaks, $\mathrm{AE}$ is the finite aggregate of edges.

Recently, Attributed Relational Graphs (ARG), Fuzzy 
Attributed Relational Graphs (FARG) and description logics have been frequently applied as image descriptions in search problems. However, the tasks to structurally analyse the objects' images and to identify similarities and differences were not assigned. Consequently, the descriptions are less structured and contain a limited number of attributes.

The proposed approach to processing, analyzing, describing and matching graph data is based on the principles and methods of system analysis and is distinguished by the methods of developing a structured, sound image description and matching the descriptions as well as the inverse links at the analysis stages aimed at providing automatic return and data specification in the image areas, defined by the subsequent stages.

\section{METHODOLOGY AND INFORMATION TECHNOLOGY OF IMAGE ANALYSIS}

The model of the process of acquiring knowledge from the semistructured data presented in fig. 1 was formed on the basis of the present-day achievements in cognitive science and system analysis. The inverse links are introduced in the given scheme with the purpose of specification.

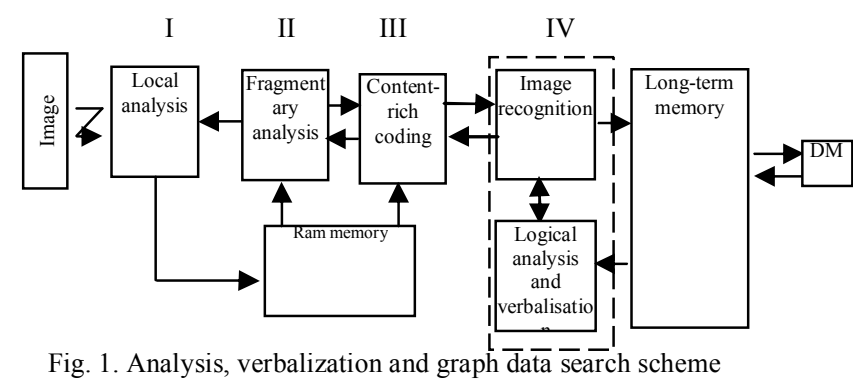

Levels I - III are the empiric cognitive levels. Level I deals with the application of evaluating and studying the image local characteristics: edge pixels, singular points and edge points. Core requirements to the methods of this group are the opportunity to immediately change the resolution and response depending on the context. In addition, integral and statistical characteristics of local area, such as average brightness and brightness distribution are calculated at this level. Inasmuch as the given level addresses every pixel of an image, it is, consequently, the most resource-intensive one. Preferably, it should be parallelized, for example, with the help of a graphics processor.

The second level focuses on analyzing object fragments, i.e. recursive optimization of singular point positions, specification of singular points (angles and branching) by means of extrapolating the beams, emanated from the singular points, tracking the edge pixel chains, tapering and other operations. Frequently, the functions of the first level are activated by addressing the image area under analysis directly. Specifically, this stage can request the change of resolving capacity or the sensitivity of a local analyzer, thus enabling to detect peculiarities, which are poorly resolved and dispersed over the area. The higher the degree of the image integration density, the lower is the resolution ability. On the other hand, the higher the resolution ability is, the more lines, bends and other noises impeding the perception of the whole picture are allocated [5]. Thus, the fast change of resolution power enables to implement abstracting and specification in the specified areas of an image quite rapidly.

Data approximation and calling (level III) complete the image syntactic structure building. The stage under consideration deals with edge approximation (by means of lines and arcs segments), color banding, skeleton eliciting, attributive graphs systematization and formation. The tasks of this level are solved by iterational approximation to the accuracy, requested by a decision maker (DM) or other processing levels.

According to the classification of the system analysis methods, level IV is theoretical. This level involves matching fuzzy attributive graphs of the image under analysis and the previously gained ones, structuring, restructuring and formalization of the descriptions on the basis of fuzzy logic. Recognition of the fuzzy attributed relational graphs lies in matching and eliciting the connected subgraphs, which have a greater degree of similarity with any subgraphs stored in the long-term memory. Image verbalization involves graph analyzing and detecting the constructions which comply with the terminological part of the knowledge data base created on the basis of description logic. In case of any disagreements in the process of drawing inductive inference, level III is addressed especially for specifying an attributive graph in the defined area of an image (color segments, degree of edges and skeleton approximation) or, even levels I or II can be addressed for changing the resolution ability and the degree of data generalization. The choice of the levels to address depends on the tasks set. The analysis outcomes are represented by semantic interpretation of syntactic structures, pragmatic measuring of the parameters, relations and trends as well as the synthesis of the objects structured descriptions made by their images.

Levels I and II rely on bioinspired techniques, and levels III and IV implement cognitive mechanisms for image recognition, inductive inference and descriptions synthesis.

An image model is formed on the level III, which is in charge of processing graphic data, as a multilayer attributive graph. Generally, graph of a layer (subject attributive graph) is a fuzzy attributed relational graph (FARG), whose peaks and edges contain quantitative and qualitative parameters. A fuzzy attributed relational graph:

$$
G=(V, A, E, R)
$$

where $\mathrm{v} \in \mathrm{V}$ is a set of conventional supporting nodes (CSN): Branch End, Branching, Angle, whose parameters a $\epsilon$ $A$ are a set of linguistic variables, taking on a value from the fuzzy sets; e $\in \mathrm{E}$ is a set of edges (nonoriented arcs), reflecting the spatial relationships between CSN with the help of parameters $r \in R$, which are a set of linguistic variables, taking the values from the fuzzy sets.

A set of attributive peaks:

$$
A=A T \cup A N \cup A L,
$$


where $A T$ are attributes, taking the text values; $A N$ are numeric attributes; $A L$ are fuzzy (linguistic) attributes.

A set of edges attributes:

$$
R=R T \cup R N \cup R L,
$$

where $R T$ are the attributes, taking the text values; $R N$ are numeric attributes; $R L$ are fuzzy (linguistic) attributes.

A set of linguistic variables $(A L)$, describing the peaks is determined by the sequence:

$$
<a, X, C a>
$$

where a is a variable title, $X$ is the scope of a variable, i.e. a set of possible values $\mathrm{x} ; C a=\{<M a(\mathrm{x}) / \mathrm{x}\rangle\}$ is a fuzzy set describing the degree to which $M a(\mathrm{x})$ of some number belongs to the linguistic value $\mathrm{x}$.

$R L=\{<r, Y, C r>\}$, a set of linguistic variables describing the edges of the graph of the object image is analogous to the set $A L$ for the peaks.

Descriptive logics have become widely used in various analytical and search systems. One of the advantages of the descriptive logics is the fact that they are used by an expert or a decision maker as the basis for describing terms and domain characterization, while the reasoner of the system controls the consistency of characterization and searches for the available examples in the data section.

The given paper suggests applying the description logic $A L C$ (Attributive Language with Complement) with the special extension to the specialized area of the data, represented as attributive graphs, let us call it $A L C(G)$, to the formal description and structural analysis of images (level IV, processing graph data) of the objects. This solution enables to narrow the domain of discourse and reduce the number of interpreting functions when solving the tasks with graphical data structure. Predicates based on geometric functions are inserted into primitive concepts for the tasks of image analyzing, while concepts syntax is complemented by settheoretic operations on image areas. It is assumed, that nowadays the tasks which are solved on the basis of graphs can be used as primitive concepts and roles when modeling the reasoning based on images.

Expanding the language $A L C(G)$ logic in the form of attributive graphs over a specific data domain reduces the scope of searching rules in the image database due to the situation when only edges chains, connecting the couples of peaks will be checked instead of checking a set of pixels $[6,7]$ or a set of segments.

As for atomic concepts, the notions interpreted in graphs and notions calculated on the image data belong to the class of non-derivative notions:

C1) Peak $(x)-x$ is a graph peak, its interpretation is a set of peaks of the graph under consideration;

C2) $\operatorname{Arc}(x)-x$ is a graph arc, its interpretation is a set of arcs of the graph under consideration;
C3) (Sub)Graph(x) - $\mathrm{x}$ is a graph's subgraph, its interpretation is a set of all subgraphs of the graph under consideration;

C4) Connected(Sub)Graph(x) - $\mathrm{x}$ is a connected subgraph of the graph under consideration;

C5) Segment( $x)-x$ is an image segment, interpretation is a set of color segments, arranged according to the principle of pixels parameters proximity in a color space.

Atomic roles, interpreted in the images graphs are as follows:

IR1) SegmentPart (x:Segment, y: Segment) - segment y is a part (subset of the pixels set) of the segment $\mathrm{x}$ - functional relation (attribute).

IR2) InsidesSegment (x: Segment, y: Segment) - segment $\mathrm{y}$ is a part (subset of the pixels set) of the segment $\mathrm{x}$ and segments limits do not intersect, i.e. all points of the limits $y$ are inside the polygon formed by the limit $\mathrm{x}$;

IR3) SegmentArea (x:Segment, $y:$ Number), $y$ is the number of pixels in segment $\mathrm{x}$;

IR4) SegmentPerimeter ( $x$ :Segment, $y:$ Number), $y$ is the length of segment x limit;

IR5) SegmentColour (x:Segment, $y:$ Number), $y$ is an average color of the segment $\mathrm{x}$ pixels;

Functional relations (attributes) characterizing the shape of segment parts are as follows:

FR1) SegmentForm (x:Segment, y:FuzzyValue) - fuzzy value $\{$ Area, Wideband, Linear\} is calculated on the basis of the ratio of the parameters area to the length.

FR2)LimitLinearity (x:SegmentLimit, y:FuzzyValue) fuzzy value $\{$ Rectilinear, Mixed, Roundish\} is calculated on the basis of the total length of the straight line segments in percentage terms to the perimeter length.

FR3) LimitsIrragularity (x:SegmentLimit, y:FuzzyValue) fuzzy value $\{$ Strong, Medium strong, Weak\} is calculated on the basis of the number of negative bends of the limits relatively to the limits length.

Some terminological principles of the subject domain "Aerospace photo interpretation" - using the notions and ratios introduced above the rules for searching objects in the images can be drawn. For example:

An aircraft is the image area whose shape is close to a polygon and the limits have 5 sharp angles or whose shape is close to a tetragon and the limits have 3 sharp angles.

$$
\begin{aligned}
\text { Aircraft } \equiv & \text { Area } \square \quad((\exists \text { hasShape } .=\text { Polygon }) \sqcap \quad(\exists \\
=5 \text { (hasAngle. }= & \text { Sharp }) \sqcup(\exists \text { hasShape } .=\text { Tetragon }) \sqcap \quad(\exists \\
& =3 \text { (hasAngle. }=\text { Sharp })) .
\end{aligned}
$$

An automobile is the image area whose shape is close to a tetragon and the area is smaller than that of an aircraft or a ship and the surrounding background is dark:

Automobile $\equiv$ Area $\sqcap \quad(\exists$ hasShape. $=$ Tetragon $) \sqcap \quad((\exists$ hasArea $<$ Aircraft hasArea $) \sqcup(\exists$ hasArea $<$ Ship hasArea $)$ 


\section{$\sqcap \quad(\exists$ hasBackground.= Dark $)$.}

A boat is the image area whose shape is close to a tetragon and is small; its limits have one or two sharp angles and a dark surrounding background.

Boat $\equiv$ Area $\sqcap \quad(\exists$ hasShape $=$ Tetragon $) \sqcap \quad(\exists$ hasArea. $=$ Small) $\sqcap \quad(\exists<3$ (hasAngle. $=$ Sharp $) \sqcap \quad(\exists$ hasBackground. $=$

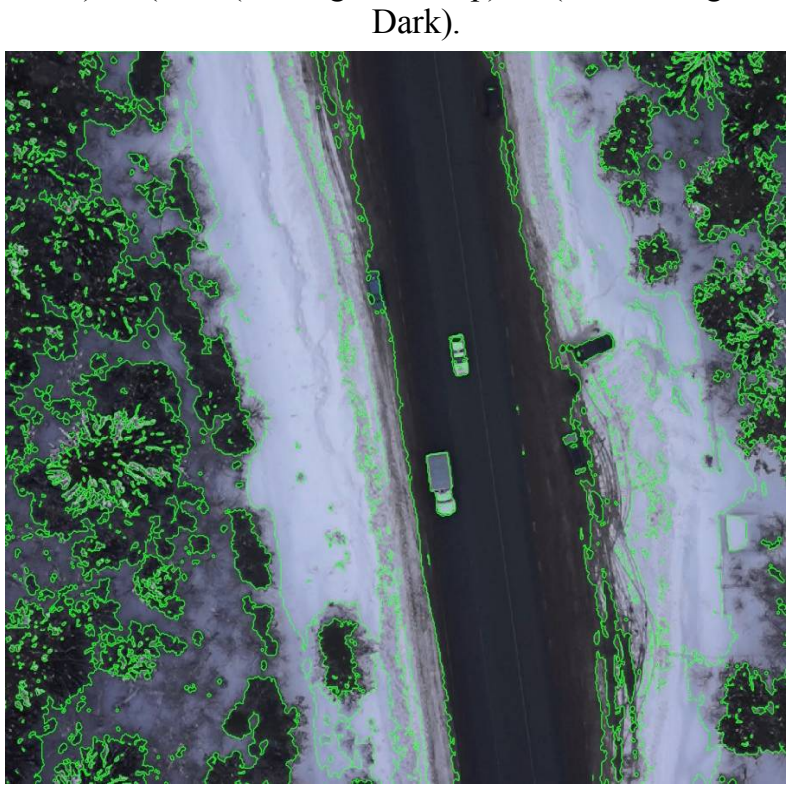

Fig. 2. Highlighting artificial objects

Afterwards, the logically described structure of the subject domains of the objects is applied for selecting specimens automatically out of the actual data by means of inductive inference and with the help of typical and specified reasoners.

\section{THE SYSTEM OF ANALYSING AN INTERPRETING AEROSPACE PHOTOS}

The approach described above was applied for creating a software package which is capable of analysing and carrying out the informal descriptions of the objects for the convenience of the decision taking in the tasks involving interpreting the aerospace photos.

The existing geo information systems (ERDAS Imagine, module ArcGIS Spatial Analyst, Complex of automated interpreting and vectoring, eCognitio, etc.) are characterized by the situation when notwithstanding a higher accuracy of the objects quantitative characteristics calculation provided by computer processing and the ability to apply digital filters for sharpening the image recognition, the volume of manual work required for interpreting aerospace photos remains to be quite considerable [8]. In modern systems of automated interpreting the image pixels are classified on the basis of either a single indicator or a set of direct interpreting ones. However, to ensure a qualitative objects' interpretation there is a need to apply the complex analysis of direct and indirect interpreting characteristics with the following inductive inferences.

The system enables to supplement the low-level characteristics (colour, texture, shape) with the quantitative and qualitative characteristics of particular homogeneous and textural domains, and the sketch generated by them; as well, owing to the model of the image presented in the form of multilevel attributive graph it makes possible to characterize spatial relationships between them, provides coordinate referencing of the photo-request and calculation of the trendshistories of the objects alterations.
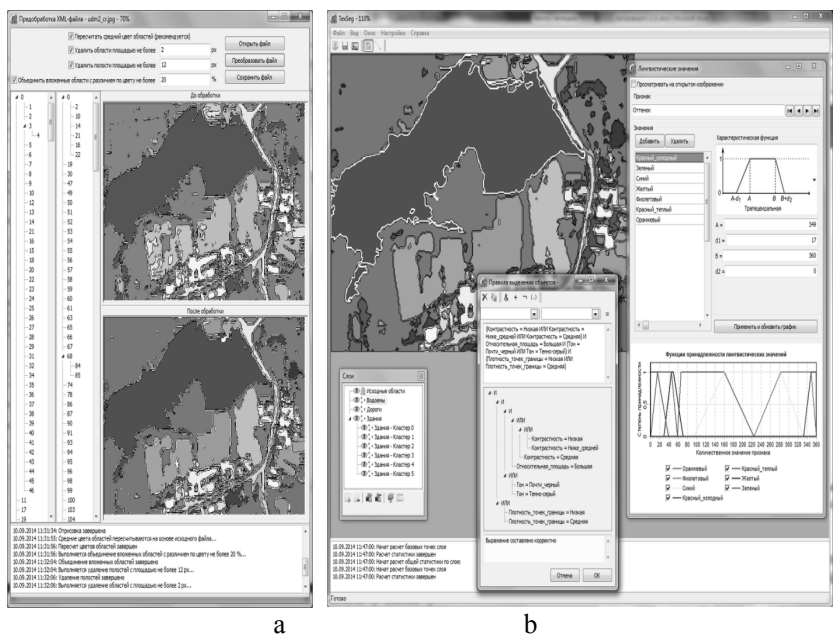

Fig. 3. Examples of semantic encoding system operation: a) the results of aerospace photos automatic vectoring; b) examples of highlighting waterbodies in the image (the popped out windows demonstrate the layers, setup of rules for the objects highlighting, setup of linguistic meanings)

Qualitative assessment of highlighting significant objects in the examples similar to the ones depicted in Fig. 2 is demonstrated in Tab. I.

TABLE I. QUALITATIVE ASSESSMENT OF HIGHLIGHTING VISUAL OBJECTS

\begin{tabular}{|l|l|l|}
\hline $\begin{array}{l}\text { Significant } \\
\text { objects }\end{array}$ & Accuracy & Completeness \\
\hline Roads & $86 \%$ & $94 \%$ \\
\hline Buildings & $82 \%$ & $85 \%$ \\
\hline Waterbodies & $83 \%$ & $90 \%$ \\
\hline Fields & $82 \%$ & $86 \%$ \\
\hline $\begin{array}{l}\text { Man-made } \\
\text { objects }\end{array}$ & $85 \%$ & $88 \%$ \\
\hline $\begin{array}{l}\text { Average } \\
\text { number }\end{array}$ & $84 \%$ & $89 \%$ \\
\hline
\end{tabular}

Accuracy of highlighting significant objects in the images is calculated according to the following formula $\left|V_{\mathrm{s}} \cap V_{\mathrm{Exp}}\right| /$ $\left|V_{\mathrm{s}}\right|$. Completeness of highlighting significant objects is calculated according to the following formula $\left|V_{\mathrm{s}} \cap V_{\text {Exp }}\right| /$ $\left|V_{\text {Exp }}\right|$, where $V_{\text {s }}$ is an array of significant objects, elicited by the system; $V_{\text {exp }}$ is an array of significant objects elicited by an expert.

\section{CONCLUSION}

Thus, considering large amounts of graph data and stiff requirements to the efficiency of solutions made on its basis, the role of system approach both to the tasks of building the 
decision support system, which uses graph data, and to the images becomes rather significant and is regarded as a semistructured system of interrelated signals. The considered approach based on cognitive principles and systems analysis methods enabled to enhance the relevance of search and accuracy of analysis and acquiring knowledge from images in the decision support tasks in engineering system.

\section{REFERENCES}

[1] Vasilieva, N. A. Metody poiska informatsii po soderzhaniyu [Methods of searching information on the basis of content], Programming. 2009, № 3, pp. 1-30.

[2] Lutsiv. V. Avtomaticheskiy analiz izobrazheniy. Obyektno nezavisimiy structurniy podhod [Autamatic image analysis. Object-independent structural approach]. Saarbrucken, Germany: Lambert Academic Publishing, 2011.

[3] Simankov V.S., Lutsenko E.V., Laptev V.N. Sistemniy analiz v adaptivnom upravlenii [System analysis in adaptive control]: Monograph (scientific publication). / Under the scientific editorship of Simankov V.S. Krasnodar: ISTE Kuban state technological university, 2001.

[4] Stotz, A., Nagi R., Sudit M. Incremental Graph Matching for Situation Awareness // 12th International Conference on Information Fusion, 2009 , pp. 452 - 459.

[5] Kuchuganov A.V. Bioincperirovannie algoritmi videleniya informativnih priznakov izobrazheniy [Bioinspired algorythms of informative features selection] // Bulletin of the Tomsk Polytechnic University 2012. Vol 321., № 5: Control, Computer Science and Technology. pp. 141-145.

[6] Zoe Falomir, Ernesto Jim' enez-Ruiz, M. Teresa Escrig, Lled' o Museros. Describing Images using Qualitative Models and Description Logics // Preprint submitted to Spatial Cognition And Computation. Taylor \& Francis. - Vol 11, Issue 1, January 2011, pp. 45-74. (http://dx.doi.org/10.1080/13875868.2010.545611).

[7] Hudelot C., Atif J. Bloch I. Alc(f): a new description logics for spatial reasoning in images // 1 st International workshop on computer vision + ontology applied cross-disciplinary technologies. 2014, pp. 1-15.

[8] Labutina I.A. Deshifrirovanie ajerokosmicheskih snimkov [Interpretation of aerospace images]. Moscow, Aspekt Press, 2004. 